\title{
ANDES

\section{Valores de normalidad de eosinófilos en mucosa gástrica y duodenal de niños referidos a endoscopía digestiva alta}

\author{
Normal values of eosinophils in gastric and duodenal mucosa of children referred \\ to upper gastrointestinal endoscopy
}

\author{
Marlene Ortiz $^{a \star}$, Francisca Jaime ${ }^{\mathrm{a}, \mathrm{e} \star}$, Loreto Ortiz ${ }^{\mathrm{b}}$, Ruby Carrasco ${ }^{\mathrm{c}, \mathrm{f}}$, María José Orellana ${ }^{\mathrm{d}}$, \\ Javiera Torres ${ }^{c}$, Andrea Villagrán ${ }^{\mathrm{d}}$, Paul R. Harris ${ }^{\mathrm{a}}$
}

\author{
aDepartamento de Gastroenterología y Nutrición Pediátrica, División de Pediatría, Facultad de Medicina, Pontificia Universidad Católica de Chile. \\ Santiago, Chile \\ bFacultad de Medicina, Universidad de Valparaíso y Hospital Comunitario Puerto Williams. Cabo de Hornos, Chile \\ 'Departamento de Anatomía Patológica, Facultad de Medicina, Pontificia Universidad Católica de Chile. Santiago, Chile \\ dFacultad de Medicina, Pontificia Universidad Católica de Chile. Santiago, Chile \\ eDepartamento de Pediatría, Clínica Alemana de Santiago-Facultad de Medicina Universidad del Desarrollo. Santiago, Chile \\ ‘Departamento de Anatomía Patológica, Complejo Asistencial Sótero del Río. Santiago, Chile \\ *Ambos autores contribuyeron por igual a este trabajo.
}

Recibido: 25 de septiembre de 2020; Aceptado: 16 de marzo de 2021

¿Qué se sabe del tema que trata este estudio?

Existe escasez de estudios amplios en población pediátrica y diversidad respecto a un punto de corte de eosinófilos (Eos) en mucosa gástrica y duodenal en individuos sanos. Se han descrito recuentos de Eos en biopsias en hemisferio norte, con escasa exploración en población latinoamericana.

\section{¿Qué aporta este estudio a lo ya conocido?}

Se describe en población pediátrica latinoamericana (chilena) recuento de Eos (promedio+1DE) en cuerpo gástrico $(n=27)$ de $1,06 \pm 1,79$ Eos/CAM (campo alto aumento), en antro gástrico $(\mathrm{n}=72)$ de $1,13 \pm 1,79$ Eos/HPF, y en duodeno $(\mathrm{n}=30) 10,44 \pm 7,09$ Eos/CAM.

\section{Resumen}

Con el aumento de la incidencia de alergias alimentarias, la presencia de eosinófilos (Eos) en la mucosa gastrointestinal ha recibido mayor atención. Sin embargo, los valores de normalidad para el recuento de Eos en estómago y duodeno en pacientes pediátricos son aún limitados. Objetivo: Estimar valores de referencia de Eos en biopsias de estómago y duodeno de niños referidos a endoscopía digestiva alta (EDA). Pacientes y Método: Estudio transversal de biopsias de niños referidos a EDA. Se analizó el informe endoscópico, test de ureasa para presencia de $H$. pylori y evaluación histológica ( $\mathrm{n}^{\circ}$ células/campo de aumento mayor o CAM). Se describe la distribución de Eos como mediana y des-
Palabras clave: Alergia Alimentaria; Eosinófilos; Mucosa Gástrica; Estómago; Helicobacter pylori

Correspondencia:

Paul R. Harris

pharris@med.puc.cl 
viación estandar, así como también la distribución percentilar, dado que los recuentos no tuvieron distribución normal. Se realizó análisis estadístico con test $\chi^{2}$, test de Wilcoxon, análisis de la varianza y curvas de regresión lineal según correspondiera. Resultados: De 170 pacientes derivados a EDA, 72 cumplían con criterios de "normalidad" (EDA normal en análisis macroscópico, test de ureasa negativo y biopsia normal). La mediana de edad fue 11 años (rango 4-16), 68\% niñas. El recuento de Eos (promedio $\pm 1 \mathrm{DE})$ en cuerpo gástrico $(\mathrm{n}=27)$ fue $1,06 \pm 1,79$ Eos/CAM, en antro gástrico $(\mathrm{n}=72)$ fue $1,13 \pm 1,79$ Eos/HPF, y en duodeno $(n=30)$ fue 10,44 \pm 7,09 Eos/CAM. No se encontraron diferencias significativas por edad y sexo, así como tampoco según infección por H. pylori $(\mathrm{p}=0,095)$. Conclusiones: Se propone como rango de referencia en niños un conteo en cuerpo gástrico de 0-3 Eos/CAM, en antro de 0-3 Eos/CAM y en duodeno de 3-17 Eos/CAM. Incluso en medios con alta prevalencia de infección por $H$. pylori, el recuento de Eos no parece ser un elemento distintivo, y éstos están presentes normalmente en la mucosa gastroduodenal.

\section{Abstract}

With the increasing incidence of food allergies, the presence of eosinophils (Eos) in the gastrointestinal mucosa has received increased attention, particularly in the esophagus and colon. However, normal values for the Eos count in the stomach and duodenum in pediatric patients are still limited. The objective of this study was to estimate Eos reference values in stomach and duodenal biopsies of children referred to upper gastrointestinal endoscopy. Patients and Methods: Cross-sectional study of biopsies from symptomatic children referred to upper gastrointestinal endoscopy. The endoscopic report, Rapid Urease Test for the presence of $H$. pylori, and the quantitative histological evaluation (number of cells/HFP, high power field) were analyzed. The Eos distribution is described as mean and standard deviation, and also as percentiles since the counts did not have a normal distribution. Statistical analysis included $\chi^{2}$ test, Wilcoxon test, analysis of variance, and linear regression curves were evaluated as appropriate. Results: Of the 170 patients referred to endoscopy, 72 met "normal" criteria (normal endoscopy in macroscopic analysis, negative Rapid Urease Test, and normal biopsy). The median age was 11 years (range $4-16$ ), and $68 \%$ were girls. The Eos count (mean $\pm 1 \mathrm{SD}$ ) in gastric antrum $(n=72)$ was $1.13 \pm 1.79 \mathrm{Eos} / \mathrm{HPF}$; in gastric body $(\mathrm{n}=27), 1.06 \pm 1.79 \mathrm{Eos} / \mathrm{HPF}$; and in duodenum $(\mathrm{n}=30), 10.44 \pm 7.09$ Eos/HPF. There were no significant differences by age and sex, or by H. pylori infection $(\mathrm{p}=0.095)$. Conclusions: We propose an Eos count of $0-3$ Eos/HPF for the gastric body, 0-3 Eos/HPF in the antrum, and 3-17 Eos/HPF in the duodenum as a normal range for gastric mucosa in children. This study suggests that in areas with a high prevalence of $H$. pylori infection, the count of Eos does not seem to be a distinctive element and that Eos are commonly present in the gastroduodenal mucosa.

\section{Keywords:}

Food Allergies;

Eosinophils;

Gastric Mucosa;

Stomach;

Helicobacter pylori

\section{Introducción}

El tracto gastrointestinal (GI) posee mayor área superficial que cualquier otro órgano en el cuerpo y contiene el mayor número de células inmunes y sus productos. Los eosinófilos (Eos) residen normalmente a lo largo de todo el tracto $\mathrm{GI}^{1}$, excepto en el esófago ${ }^{2}$, y hay una gradiente ascendente en el recuento de cefálico a caudal, con mayores niveles en ciego que en estómago $^{3,4}$. Los eosinófilos son granulocitos multifuncionales involucrados en alergias, infecciones parasitarias, en reacciones de hospedero-tumor ${ }^{5,6}$ y también ayudan a mantener la homeostasis intestinal epitelial ${ }^{2,4,7}$. Los Eos pueden interactuar regulando la microbiota intestinal, especialmente en vista del efecto antibacteriano de sus proteínas granulares y la capacidad de modificar la inmunidad innata $a^{5,8}$.
En las últimas décadas, el rol de los Eos ha sido estudiado, en particular su papel en la mantención de la salud o el desarrollo de enfermedades del tracto GI. Los desórdenes gastrointestinales asociados a eosinófilos (eosinophil-associated gastrointestinal disorders o EGIDs, por su sigla en inglés) abarcan una serie de enfermedades, caracterizadas por inflamación eosinofílica en distintos segmentos del tubo GI, en ausencia de otras causas conocidas de eosinofilia9 ${ }^{9}$ Los EGIDs incluyen la esofagitis eosinofílica (EEo), la gastritis eosinofílica (GEo), la gastroenteritis eosinofílica (GEEo) y la colitis eosinofílica $(\mathrm{CEO})^{4,8,10}$. Sin embargo, la mayor parte de la información conocida corresponde a la EEo $^{11,12}$ siendo el resto consideradas de presentación clínica muy escasa ${ }^{13,14}$. Es por esto, que a pesar de los avances tecnológicos, aún no se ha logrado establecer de forma clara los criterios diagnósticos para va- 
rias EGIDs ${ }^{15-21}$. La gastritis eosinofílica es una entidad mucho más rara, comparada con la EEo. Existe escasez de estudios amplios en población pediátrica y no existe consenso respecto a un punto de corte de Eos en mucosa gástrica y duodenal en individuos sanos ${ }^{4,9}$, menos aún en población pediátrica ${ }^{6,7,22-24}$. En los estudios disponibles, existe mucha variabilidad del umbral en estómago considerado como diagnóstico por los distintos autores: desde 20 Eos/campo de aumento mayor o CAM, 30 Eos en al menos 5 CAM, hasta algunos que consideran 80 Eos/CAM ${ }^{8}$.

De lo disponible a la fecha, en cuanto a valores de normalidad de Eos en mucosa gástrica, se han descrito recuentos en biopsias de población pediátrica en el hemisferio norte ${ }^{15,16,21}$, con escasa exploración en población latinoamericana ${ }^{11}$.

El objetivo fue estimar valores de referencia de eosinófilos en biopsias de estómago y duodeno de niños chilenos referidos a endoscopía digestiva alta por síntomas digestivos habituales (dolor abdominal crónico, DAC), cuyo resultado macroscópico e histológico fue normal.

\section{Pacientes y Método}

Se utilizaron los análisis histológicos provenientes de 2 bases de datos de estudios previos, ambos estudios de diseño transversal, con similares criterios de inclusión y exclusión: pacientes menores de 18 años, referidos a endoscopía digestiva alta, en su mayoría por dolor abdominal crónico. De ambas bases, se seleccionaron aquellos pacientes en los cuales se contaba con el informe endoscópico (cuyo resultado se categorizó como: normal, gastropatía, nodularidad antral, duodenopatía, enfermedad por úlcera gastroduodenal y otros), el resultado del test rápido de ureasa para determinar presencia de Helicobacter pylori (H. pylori) (positivo o negativo), y el análisis histopatológico.

\section{Recuento de eosinófilos}

Las muestras de biopsia se sometieron a fijación con formalina al 10\% v/v (volumen/volumen) tamponada a $\mathrm{pH} 7,4$ por 24 a $36 \mathrm{~h}$, y posteriormente se procesaron con técnicas histológicas de rutina para embeberlas en parafina con el fin de obtener tacos de inclusión. A partir de estos tacos se realizaron cortes de $5 \mu \mathrm{m}$ en micrótomo rotatorio para obtener placas histológicas, las que posteriormente fueron desparafinadas y teñidas con hematoxilina y eosina (HE). Después de este procesamiento, las placas se analizaron al microscopio óptico de luz.

Una anatomopatóloga realizó evaluación cuantitativa de Eos en mucosa (células/CAM) considerando el campo de aumento mayor a aquel con un aumento de $400 x$, equivalente a $0,237 \mathrm{~mm}^{2}$, en cada localización (antro, cuerpo y duodeno, cuando estuvo disponible), considerando "satisfactorias" aquellas muestras que cumplieran los siguientes criterios:

- Contener al menos el 75\% del campo con células.

- Luego de ser procesadas a láminas para análisis microscópico, permitieron la evaluación de al menos 5 CAM.

- Se logró reconocer características propias de antro, cuerpo gástrico o duodeno según fuese el caso.

Se consideraron como Eos aquellos en que se reconociese núcleo, asociado a gránulos eosinofílicos agrupados alrededor de éste (figura 1). El recuento de eosinófilos se realizó en la mucosa de tejido digestivo a nivel de la lámina propia.

La anatomopatóloga fue ciega respecto de los datos clínicos de dichos niños y de la presencia de H. pylori por test rápido de ureasa.

\section{Análisis de datos}

Para el análisis estadístico los datos se ingresaron a una base de datos Excel y fueron analizados con el programa STATA 12. Se evaluó: i) recuento de Eos en cuerpo gástrico, antro, y duodeno; ii) recuento de Eos según resultado de endoscopía y iii) para aquellas endoscopías sin hallazgos patológicos (EDA normal, $H$. pylori por test de ureasa negativo y biopsia normal), se evaluó el recuento de Eos según edad y sexo del paciente. Para cada paciente, se consideró el número de Eos por sitio, como el promedio por al menos 5 CAM. Se obtuvo el promedio del conteo y 1 desviación estándar (DE), considerando lo descrito en otras publicaciones.

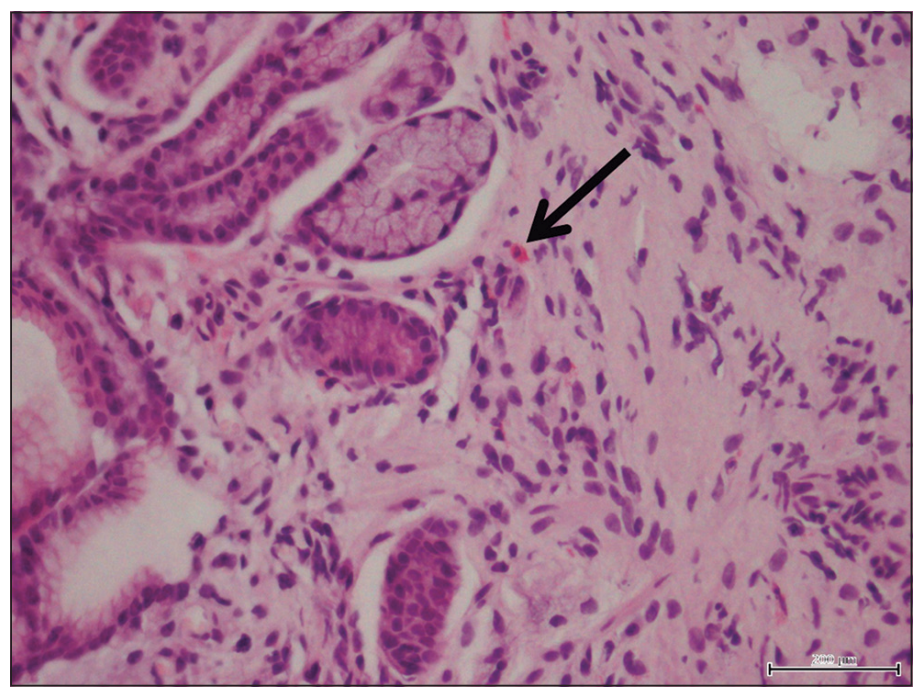

Figura 1. Ejemplo de campo de aumento mayor (CAM, 400x) para conteo de eosinófilos en mucosa gástrica. La flecha indica un eosinófilo, reconociendo en éste su núcleo y citoplasma con acúmulo de gránulos eosinofílicos alrededor. 
Sin embargo, dado que las variables edad y recuento de Eos no tuvieron distribución normal, se incluyó la descripción de los percentiles 5-25-50 (mediana)-75-95.

El análisis respecto a diferencias entre variables cualitativas, se realizó con test $\chi^{2}$, y los parámetros cuantitativos fueron comparados por test de Wilcoxon. Se realizó análisis de la varianza para estudio entre variables cualitativas y cuantitativas. También se realizaron curvas de regresión lineal para estudio entre variables cuantitativas (conteo de Eos en los diferentes tractos según edad).

\section{Aspectos éticos}

Tanto los informes endoscópicos como las muestras analizadas para este estudio fueron obtenidos en proyectos de investigación realizados y aprobados previamente por el Comité de Ética de la Facultad de Medicina de la Pontificia Universidad Católica de Chile (No 0145-05 y No 09-099), junto a sus respectivos consentimientos informados. El acceso a la base de datos fue de manera codificada y anonimizada para datos personales de los participantes.

\section{Resultados}

\section{Pacientes}

Se analizaron las biopsias de 170 pacientes. La edad no tuvo distribución normal, con una mediana de 12 años (rango 3-16 años), de los cuales 99 fueron niñas

\begin{tabular}{|c|c|c|}
\hline & Percentil & $\begin{array}{l}\text { Grupo endoscopía normal, } \\
\text { test de ureasa normal y biopsia } \\
\text { normal, recuento Eos/CAM }\end{array}$ \\
\hline \multirow[t]{5}{*}{ Cuerpo } & p5 & 0 \\
\hline & p25 & 0 \\
\hline & p50 & 0,6 \\
\hline & p75 & 1,3 \\
\hline & p95 & 2,7 \\
\hline \multirow[t]{5}{*}{ Antro } & p5 & 0 \\
\hline & p25 & 0 \\
\hline & p50 & 0,6 \\
\hline & p75 & 1,4 \\
\hline & p95 & 5 \\
\hline \multirow[t]{5}{*}{ Duodeno } & p5 & 2,6 \\
\hline & p25 & 5,4 \\
\hline & p50 & 8,6 \\
\hline & p75 & 13,5 \\
\hline & p95 & 22,5 \\
\hline
\end{tabular}

Eos/CAM= eosinofilos/campo de aumento mayor.
$(58,2 \%)$. Se obtuvo 72 biopsias disponibles para análisis de cuerpo gástrico, 170 biopsias para análisis de antro gástrico, y 72 muestras para análisis de segunda porción duodenal. De estas muestras, se consideraron satisfactorias 66 muestras de cuerpo gástrico, 167 de antro y 68 de duodeno.

\section{Conteo de eosinófilos en población pediátrica con EDA normal, biopsia normal y test rápido de ureasa negativo para detección de $\boldsymbol{H}$. pylori}

De los 170 pacientes derivados a EDA, 72 cumplieron con los criterios mencionados de normalidad. En este grupo, la mediana de edad fue 11 años (rango 4-16), 49 fueron mujeres $(68 \%)$. El recuento de eosinófilos no mostró distribución normal (test de Shapiro-Wilk, $\mathrm{p}<0,001)$. El recuento promedio $\pm \mathrm{DE}$ en cuerpo gástrico (27 muestras) fue 1,06 $\pm 1,79 \mathrm{Eos} / \mathrm{HPF}$, en antro gástrico (71 muestras) fue de 1,13 $\pm 1,79$ Eos/HPF, y en duodeno (30 muestras) de 10,44 \pm 7,09 Eos/HPF (distribución percentilar del grupo "normal", en Tabla 1). Si consideramos como límite superior de normalidad el percentil 95, los valores límites fueron 2,7, 5 y 22,5, para cuerpo gástrico, antro gástrico y duodeno, respectivamente (tabla 1 ).

$\mathrm{Al}$ analizar por sexo y edad, no se encontraron diferencias significativas.

\section{Conteo de eosinófilos en población pediátrica con EDA alterada}

De los 170 pacientes derivados a EDA, 76 presentaron alguna alteración macroscópica de ésta. En este grupo de pacientes, la mediana de edad fue 13 años (rango 3-16), 37 niñas (48,7\%). En la tabla 2 se muestra un desglose de los hallazgos macroscópicos en la EDA alterada, con el respectivo conteo de Eos/CAM.

\section{Conteo de eosinófilos en población pediátrica según test rápido de ureasa}

De la muestra total, 54 pacientes tuvieron test de ureasa positivo $(31,8 \%)$. No se encontró una diferencia significativa para el conteo de Eos/CAM en antro ni cuerpo gástrico ni en duodeno, entre la población con test de ureasa positivo y negativo $(\mathrm{p}=0,095)$ (tabla 3).

\section{Discusión}

En el presente trabajo, se encontró un recuento promedio de eosinófilos en mucosa de cuerpo gástrico de 1,06 \pm 1,79 Eos/CAM (percentil 5-95: 0-2,7); en antro gástrico de 1,13 \pm 1,79 Eos/CAM (percentil 5-95: $0-5$ ) y en duodeno de 10,44 \pm 7,09 Eos/CAM (percentil 5-95: 2,6-22,5), en niños con EDA y biopsias normales y test rápido de ureasa negativo. Esta información es similar a la entregada por otros grupos de estudio en 
Tabla 2. Distribución del recuento de eosinófilos en la muestra estudiada, en pacientes con endoscopía alterada.

\begin{tabular}{|c|c|c|c|c|c|c|c|c|c|c|}
\hline \multirow[b]{2}{*}{ Hallazgo endoscópico } & \multirow[b]{2}{*}{$\begin{array}{c}\text { Pacientes } \\
\mathrm{n}\end{array}$} & \multicolumn{3}{|c|}{ Cuerpo } & \multicolumn{3}{|c|}{ Antro } & \multicolumn{3}{|c|}{ Duodeno } \\
\hline & & $\begin{array}{c}\text { Promedio } \\
\pm D E\end{array}$ & $\begin{array}{l}\text { Mediana } \\
\text { (p25-75) }\end{array}$ & $n^{*}$ & $\begin{array}{c}\text { Promedio } \\
\pm D E\end{array}$ & $\begin{array}{l}\text { Mediana } \\
(\text { p25-75) }\end{array}$ & $n^{*}$ & $\begin{array}{c}\text { Promedio } \\
\pm D E\end{array}$ & $\begin{array}{l}\text { Mediana } \\
(\text { p25-75) }\end{array}$ & $n$ * \\
\hline Gastropatía & 11 & $1 \pm 0,70$ & $0,7(0,6-1,2)$ & 6 & $2,7 \pm 2,8$ & $1,4(0,7-3,8)$ & 11 & $9,0 \pm 4,9$ & $7,4(6,9-11,2)$ & 6 \\
\hline Nodularidad antral & 30 & $2,4 \pm 2,0$ & $1,9(1,1-3)$ & 12 & $2,2 \pm 3,8$ & $0,7(0,2-2,7)$ & 30 & $8 \pm 4,9$ & $7(4,8-12,5)$ & 11 \\
\hline Duodenopatía & 7 & $3,8 \pm 3,0$ & $4,3(0,6-6,6)$ & 3 & $1,7 \pm 3,3$ & $0,2(0,1-1,1)$ & 7 & $7,0 \pm 2,4$ & $7,4(4,4-9,2)$ & 3 \\
\hline Úlcera gastroduodenal & 11 & ND & & 0 & $2,2 \pm 2,3$ & $1,2(0,5-3,2)$ & 11 & ND & & 0 \\
\hline Otros diagnósticos & 17 & $1,3 \pm 2,0$ & $0,6(0,1-1,7)$ & 8 & $1,8 \pm 1,6$ & $1,2(0,8-2,2)$ & 17 & $20,5 \pm 33$ & $10,7(6,6-12,8)$ & 10 \\
\hline Total & 76 & & & 29 & & & 76 & & & 30 \\
\hline
\end{tabular}

$\mathrm{n}^{*}=$ número de biopsias con muestra satisfactoria. $\mathrm{n}=$ número de pacientes. $\mathrm{DE}=$ desviación estándar. $\mathrm{p}=$ percentil. $\mathrm{ND}=\mathrm{no}$ disponible.

Tabla 3. Comparación en el recuento de eosinófilos en antro gástrico según test de ureasa (ausencia/presencia de $\boldsymbol{H}$. pylori)

\begin{tabular}{ccc}
\hline Grupo & Frecuencia (pacientes con muestras & Promedio Eos/CAM \pm DE \\
& analizables de antro)
\end{tabular}

Test de ureasa negativo (ausencia H. pylori)

$113 \quad 1,50 \pm 2,17$

Test de ureasa positivo (presencia de H. pylori)

Eos/CAM = eosinofilos/campo de aumento mayor. DE = desviación estándar.

poblaciones pediátricas distintas a la nuestra, como el de DeBrosse et al. ${ }^{21}$, Lowichik et al. ${ }^{15}$ y Kalach et al. ${ }^{16}$, aunque menor que el realizado por Chernetsova et al., que utilizó otra tinción y definición de $\mathrm{CAM}^{25}$. En particular, en el estudio de DeBrosse et al., que es uno de los iniciales y más citados, se reevaluaron biopsias del tracto GI de 28 niños en EE.UU., previamente interpretadas como "sin anormalidades diagnósticas", encontrándose $1,9 \pm 1,3$ y $2,1 \pm 2,4$ Eos/CAM $\pm \mathrm{DE}$ en la lámina propia de antro y fondo gástrico, respectivamente, y 9,6 \pm 5,3 Eos/CAM \pm DE para duodeno ${ }^{21}$.

En Chile, la información disponible en población pediátrica corresponde a un estudio de marcadores de alergias alimentarias ${ }^{26}$. Un $74,1 \%$ de los pacientes sin hipersensibilidad alimentaria (HA) presentó Eos en la mucosa gástrica comparado con un $100 \%$ en el grupo con HA, constituyendo una diferencia significativa, pero no se observó diferencia significativa en el recuento de eosinófilos entre ambos grupos: $0,3 \pm 0,4$ Eos/ CAM en el grupo sin HA y $0,63 \pm 1,18$ eos/CAM en el grupo con HA.

Nuestros datos, relevantes para una población pediátrica latina no analizada previamente, confirman la aparición de una gradiente con un aumento del conteo de Eos en mucosa digestiva de proximal a distal. Lo anterior podría ser interpretado, de forma paralela, a la digestión de alimentos, en que de cefálico a caudal aumenta el contacto de la mucosa GI con productos cada vez más digeridos, aumentando también la densidad intraluminal de bacterias ${ }^{16}$.

No encontramos diferencias por sexo ni edad en el conteo de Eos, similar a lo descrito por Lowichik et al., DeBrosse et al. y Lwin et al. ${ }^{17}$.

Por otro lado, en nuestro país destaca la alta seroprevalencia de $H$. pylori en la población chilena adulta, con $73 \%$ positividad ${ }^{27}$. En niños chilenos la prevalencia varía desde $18,1 \%^{28}$, hasta un $55,9 \%$ dependiendo del nivel socioeconómico evaluado ${ }^{29}$. En nuestro estudio no se detectó asociación entre el conteo de Eos y $H$. pylori, similar a lo reportado por Lwin et al. Sin embargo, esto es diferente a lo descrito por Kalach et al. y Ashorn et al. ${ }^{30} \mathrm{y}$ a otros estudios en adultos ${ }^{6}$.

Recientemente, en un estudio en niños de Corea del Sur ${ }^{31,32}$, se realizó conteo de Eos en mucosa gástrica, duodenal, y colónica, encontrando una diferencia significativa, con mayor recuento en antro gástrico y duodeno en la población con desórdenes funcionales del tracto GI (según clasificación de Roma III en el estudio 2016, con 105 pacientes, y posterior actualización a Roma IV con estudio en 2018 de 56 pacientes), sugiriendo un rol de estas células en la fisiopatología de los trastornos funcionales, lo que no fue evaluado en este trabajo.

Nuestro estudio y los previamente citados comparten limitantes, ya que incluyen niños sintomáticos digestivos referidos a EDA (la mayoría realizadas para 
estudio de dolor abdominal crónico), lo que dificulta hablar de "normalidad" y por lo tanto, en este estudio lo nombramos como "referencia". Este estudio se basó en biopsias de niños con DAC, con hallazgo macroscópicos y microscópicos normales. Probablemente este grupo de pacientes es lo mas cercano a lo que podríamos llamar "niños sanos o normales". El realizar estudios histológicos en tubo digestivo tiene limitantes éticas en población pediátrica sana, ya que se utilizan métodos invasivos para ello.

En algunos estudios, se menciona que la utilización únicamente de hematoxilina y eosina (HE) para la tinción de las muestras sería una limitante para el análisis histológico, ya que podría subestimar el número de Eos presente en cada análisis ${ }^{16}$, proponiéndose el uso de tinción de Luna para gránulos de eosinófilos (técnica histoquímica específica para los gránulos de Eos, mejorando la detección histológica de estos granulocitos). Sin embargo, la mayoría de las experiencias alrededor del mundo han sido realizadas con tinción con $\mathrm{HE}$, con resultados bastante consistentes, como se detalló mas arriba.

En conclusión, nosotros proponemos como rango de referencia para mucosa gástrica en niños, un conteo de Eos en cuerpo de 0-3 Eos/CAM, en antro de 0-3 Eos/CAM y duodeno de 3-17 Eos/CAM. Los Eos están presentes normalmente en la mucosa gastroduodenal. El establecimiento de puntos de corte de referencia, en edades precoces, universalmente validados en diferentes áreas geográficas, es una necesidad para el clínico, y este trabajo es un aporte significativo a esta área de creciente desarrollo.

\section{Responsabilidades Éticas}

Protección de personas y animales: Los autores declaran que los procedimientos seguidos se conformaron a las normas éticas del comité de experimentación humana responsable y de acuerdo con la Asociación Médica Mundial y la Declaración de Helsinki.

Confidencialidad de los datos: Los autores declaran que han seguido los protocolos de su centro de trabajo sobre la publicación de datos de pacientes.

Derecho a la privacidad y consentimiento informado: Los autores han obtenido el consentimiento informado de los pacientes y/o sujetos referidos en el artículo. Este documento obra en poder del autor de correspondencia.

\section{Conflicto de intereses}

Los autores declaran no tener conflicto de intereses.

\section{Financiamiento}

Proyecto Fondecyt N¹130387, Chile.

\section{Agradecimientos}

Diego Romero Vera, Encargado de Biobanco y Proyectos, Departamento de Anatomía Patológica, Facultad de Medicina, Pontificia Universidad Católica de Chile.

\section{Referencias}

1. Mehta P, Furuta GT. Eosinophils in Gastrointestinal Disorders: Eosinophilic Gastrointestinal Diseases, Celiac Disease, Inflammatory Bowel Diseases, and Parasitic Infections. Immunol Allergy Clin North Am. 2015;35(3):413-37. doi: 10.1016/j.iac.2015.04.003.

2. Powell N, Walker MM, Talley NJ. Gastrointestinal eosinophils in health, disease and functional disorders. Nat Rev Gastroenterol Hepatol. 2010;7(3):146-56. doi: 10.1038/nrgastro.2010.5.

3. Pensabene L, Brundler MA, Bank JM, Di Lorenzo C. Evaluation of mucosal eosinophils in the pediatric colon. Dig Dis Sci. 2005;50(2):221-9. doi: 10.1007/ s10620-005-1586-0.

4. Aceves S, Hirano I, Furuta GT, Collins $\mathrm{MH}$. Eosinophilic gastrointestinal diseases--clinically diverse and histopathologically confounding. Semin Immunopathol. 2012;34(5):715-31. doi: 10.1007/s00281-012-0324-x.

5. Jung Y, Rothenberg ME. Roles and regulation of gastrointestinal eosinophils in immunity and disease. J Immunol. 2014;193(3):999-1005. doi: 10.4049/ jimmunol.1400413.

6. Ieni A, Barresi V, Rigoli L, Fedele F, Tuccari G, Caruso RA. Morphological and Cellular Features of Innate Immune Reaction in Helicobacter pylori Gastritis: A Brief Review. Int J Mol Sci. 2016;17(1):109. doi: 10.3390/ ijms17010109.

7. Weller PF, Spencer LA. Functions of tissue-resident eosinophils. Nat Rev Immunol. 2017;17(12):746-60. doi: 10.1038/nri.2017.95.

8. Ko HM, Morotti RA, Yershov O, Chehade M. Eosinophilic gastritis in children: clinicopathological correlation, disease course, and response to therapy. Am J Gastroenterol. 2014;109(8):1277-85. doi: 10.1038/ajg.2014.166.

9. Alhmoud T, Hanson JA, Parasher
G. Eosinophilic Gastroenteritis: An Underdiagnosed Condition. Dig Dis Sci. 2016;61(9):2585-92. doi: 10.1007/s10620016-4203-5.

10. Cherian S, Smith NM, Forbes DA. Rapidly increasing prevalence of eosinophilic oesophagitis in Western Australia. Arch Dis Child. 2006;91(12):1000-4. doi: 10.1136/adc.2006.100974.

11. Kapel RC, Miller JK, Torres C, Aksoy S, Lash R, Katzka DA. Eosinophilic esophagitis: a prevalent disease in the United States that affects all age groups. Gastroenterology.2008;134(5):1316-21. doi: 10.1053/j.gastro.2008.02.016.

12. Shah A, Kagalwalla AF, Gonsalves N, Melin-Aldana H, Li BU, Hirano I. Histopathologic variability in children with eosinophilic esophagitis. Am J Gastroenterol. 2009;104(3):716-21. doi: 10.1038/ajg.2008.117.

13. Reed C, Woosley JT, Dellon ES. Clinical characteristics, treatment outcomes, and resource utilization in children and 
adults with eosinophilic gastroenteritis. Dig Liver Dis. 2015;47(3):197-201. doi: 10.1016/j.dld.2014.11.009.

14. Busoni VB, Lifschitz C, Christiansen S, G de Davila MT, Orsi M. Gastroenteropatía eosinofílica: una serie pediátrica [Eosinophilic gastroenteropathy: a pediatric series]. Arch Argent Pediatr. 2011;109(1):68-73. doi: 10.1590/S032500752011000100019.

15. Lowichik A, Weinberg AG. A quantitative evaluation of mucosal eosinophils in the pediatric gastrointestinal tract. Mod Pathol. 1996;9(2):110-4.

16. Kalach N, Huvenne H, Gosset $\mathrm{P}$, et al. Eosinophil counts in upper digestive mucosa of Western European children: variations with age, organs, symptoms, Helicobacter pylori status, and pathological findings [published correction appears in J Pediatr Gastroenterol Nutr. 2018;66(5):843. J Pediatr Gastroenterol Nutr. 2011;52(2):175-82. doi: 10.1097/ MPG.0b013e3181e2ae00.

17. Lwin T, Melton SD, Genta RM. Eosinophilic gastritis: histopathological characterization and quantification of the normal gastric eosinophil content. Mod Pathol. 2011;24(4):556-63. doi: 10.1038/ modpathol.2010.221.

18. Ammoury RF, Rosenman MB, Roettcher D, Gupta SK. Incidental gastric eosinophils in patients with eosinophilic esophagitis: do they matter?. J Pediatr Gastroenterol Nutr. 2010;51(6):723-6. doi: 10.1097/MPG.0b013e3181d98e6c

19. Yantiss RK. Eosinophils in the GI tract: how many is too many and what do they mean?. Mod Pathol. 2015;28 Suppl 1:S7S21. doi: 10.1038/modpathol.2014.132.
20. Collins MH, Capocelli K, Yang GY. Eosinophilic Gastrointestinal Disorders Pathology. Front Med (Lausanne). 2018;4:261. doi: 10.3389/ fmed.2017.00261.

21. DeBrosse CW, Case JW, Putnam PE, Collins MH, Rothenberg ME. Quantity and distribution of eosinophils in the gastrointestinal tract of children. Pediatr Dev Pathol. 2006;9(3):210-8. doi: 10.2350/11-05-0130.1.

22. Koutri E, Papadopoulou A. Eosinophilic Gastrointestinal Diseases in Childhood. Ann Nutr Metab. 2018;73 Suppl 4:18-28. doi: $10.1159 / 000493668$.

23. Jensen ET, Martin CF, Kappelman MD, Dellon ES. Prevalence of Eosinophilic Gastritis, Gastroenteritis, and Colitis: Estimates from a National Administrative Database. J Pediatr Gastroenterol Nutr. 2016;62(1):36-42. doi: 10.1097/ MPG.0000000000000865.

24. Zevit N, Furuta GT. Eosinophilic Gastroenteritis and Colitis: Not Yet Ready for the Big Leagues. J Pediatr Gastroenterol Nutr. 2018;67(1):1-2. doi: 10.1097/MPG.0000000000001998.

25. Chernetsova E, Sullivan K, de Nanassy J, et al. Histologic analysis of eosinophils and mast cells of the gastrointestinal tract in healthy Canadian children. Hum Pathol. 2016;54:55-63. doi: 10.1016/j. humpath.2016.03.004.

26. Talesnik GE, Majerson GD, Serrano HC, et al. Marcadores de alergia alimentaria en enfermedad péptica. Rev Chil Pediatr. 2009;80(2): 121-8.

27. Ferreccio C, Rollán A, Harris PR, et al. Gastric cancer is related to early Helicobacter pylori infection in a high- prevalence country. Cancer Epidemiol Biomarkers Prev. 2007;16(4):662-7. doi: 10.1158/1055-9965.EPI-06-0514.

28. Jaime F, Villagrán A, Serrano C, Cerda J, Harris PR. Prevalencia de la infección por Helicobacter pylori en niños: estimando la edad de adquisición [Frequency of Helicobacter pylori infection in 144 school age Chilean children]. Rev Med Chile 2013;141(10):1249-54. doi: 10.4067/ S0034-98872013001000003.

29. Jaime F, Villagrán A, Hernández C, Ortiz M, Serrano C, Harris PR. Functional gastrointestinal disorders in children from low socio-economic status and Helicobacter pylori infection. Child Care Health Dev. 2018;44(2):319-25. doi: 10.1111/cch.12486.

30. Ashorn M, Ruuska T, Karikoski R, Välipakka J, Mäki M. Gastric mucosal cell densities in Helicobacter pyloripositive and -negative dyspeptic children and healthy controls. J Pediatr Gastroenterol Nutr. 1994;18(2):146-51. doi: 10.1097/00005176-199402000-00005.

31. Lee EH, Yang HR, Lee HS. Analysis of Gastric and Duodenal Eosinophils in Children with Abdominal Pain Related Functional Gastrointestinal Disorders According to Rome III Criteria. J Neurogastroenterol Motil. 2016;22(3):459-69. doi: 10.5056/ jnm15174.

32. Lee EH, Yang HR, Lee HS. Quantitative Analysis of Distribution of the Gastrointestinal Tract Eosinophils in Childhood Functional Abdominal Pain Disorders. J Neurogastroenterol Motil. 2018;24(4):614-27. doi: 10.5056/ jnm18050. 